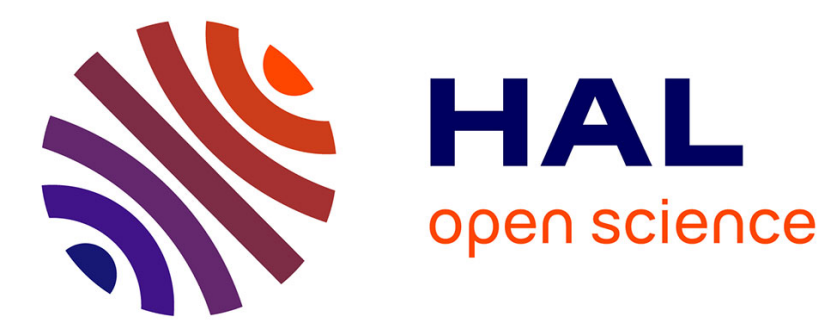

\title{
Qualité de la litière en aviculture. Aliments et caractéristiques physiques des excretas
}

\author{
B. Carré, F. de Monredon, J.-P. Melcion, J. Gomez
}

\section{To cite this version:}

B. Carré, F. de Monredon, J.-P. Melcion, J. Gomez. Qualité de la litière en aviculture. Aliments et caractéristiques physiques des excretas. Productions Animales, 1995, 8 (5), pp.331-334. hal-00896129

\section{HAL Id: hal-00896129 \\ https://hal.science/hal-00896129}

Submitted on 1 Jan 1995

HAL is a multi-disciplinary open access archive for the deposit and dissemination of scientific research documents, whether they are published or not. The documents may come from teaching and research institutions in France or abroad, or from public or private research centers.
L'archive ouverte pluridisciplinaire HAL, est destinée au dépôt et à la diffusion de documents scientifiques de niveau recherche, publiés ou non, émanant des établissements d'enseignement et de recherche français ou étrangers, des laboratoires publics ou privés. 
INRA Prod. Anim., $1995,8(5), 331-334$
B. CARRÉ, F. DE MONREDON*, J.-P. MELCION*, J. GOMEZ

INRA Station de Recherches Avicoles 37380 Nouzilly

* INRA Laboratoire de Technologie Appliquée à la Nutrition, BP 527, 44026 Nantes Cedex

\section{Qualité de la litière en aviculture. Aliments et caractéristiques physiques des excretas}

La qualité des litières des oiseaux d'élevage en croissance dépend en
partie des caractéristiques physiques des excretas. Cette étude montre
comment on peut améliorer le contrôle de ces caractéristiques en
maîtrisant la composition de l'aliment et son traitement technologique.

Les systèmes de productions avicoles requièrent un contrôle soigneux de la qualité des litières d'élevage. Cette dernière se définit en partie par les caractéristiques physiques des excretas, en raison de leur influence sur les facilités de manutention des déjections, sur les risques d'apparition de lésions aux pattes et au bréchet, et sur la répartition spatiale (air, surface de litière, profondeur de litière) de l'humidité dans l'élevage. Ces caractéristiques physiques peuvent également intervenir dans l'apparence visuelle des litières, facteur capable d'influen- cer le jugement de l'éleveur sur la qualité de l'aliment.

Cet article complète l'étude publiée précédemment dans cette revue (Carré et al 1994), dans laquelle avaient été présentés les facteurs alimentaires influençant les consommations et excrétions d'eau chez le dindonneau de chair en croissance. Les résultats présentés ici concernent les propriétés de collage des excretas et leur note visuelle de déstructuration.

\section{Conditions expérimentales}

\section{Résumé}

L'article décrit la suite d'une expérience dans laquelle avait été établie la prédiction des consommations et excrétions d'eau chez les dindonneaux de chair. Les résultats présentés ici sont relatifs à la prédiction de l'état physique des excretas. Les excretas de dindonneaux nourris avec 27 régimes granulés différents sont caractérisés pour leur pouvoir collant à l'aide d'un rhéomètre destiné aux matériaux pâteux. Des notes visuelles de déstructuration sont également attribuées aux excretas.

Le pouvoir collant et la note de déstructuration des excretas sont positivement corrélés entre eux $\left(R^{2}=0,26 ; n=216\right)$ et sont chacun positivement corrélés à la teneur en eau des excretas $\left(R^{2}=0,23\right.$ et $R^{2}=0,55$, respectivement; $\left.n=216\right)$. Ces mesures (valeurs moyennes par régime) sont reliées aux caractéristiques des régimes par régressions linéaires multiples. Les meilleures équations comportent les deux variables "viscosité utile réelle " (VUR) et " eau retenue par les parois végétales " (EauPAR). Les variables VUR et EauPAR sont respectivement affectées de coefficients positifs et négatifs. Les $R^{2}$ des régressions sont de 0,485 et 0,819 pour le pouvoir collant et la note de déstructuration des excretas, respectivement. La rétention d'eau des parois végétales des régimes dépend de la rétention d'eau des parois des matières premières utilisées, qui varie dans un rapport de 1 à 4, et de la température en sortie de presse. Les plus fortes températures entraînent une réduction du pouvoir de rétention d'eau des parois végétales.
L'expérience est celle décrite dans l'article de Carré et al (1994). Au 34 ${ }^{\mathrm{e}}$ jour d'âge, les excretas sont collectés individuellement pendant $24 \mathrm{~h}$, notés (de 1 à 5) par 3 personnes pour leur état de déstructuration, puis homogénéisés et stockés à $+4{ }^{\circ} \mathrm{C}$ pendant $48 \mathrm{~h}$, et, enfin, mesurés pour leur propriété collante.

La note visuelle de déstructuration des excretas est établie d'une manière croissante (de 1 à 5) avec l'état apparent de déstructuration, les notes les plus basses correspondant aux excretas les plus fermes et les plus homogènes, les notes les plus élevées concernant les fientes les plus liquides d'aspect. Une moyenne provenant des 3 notateurs est attribuée à chaque animal.

La propriété collante des excretas est déterminée à l'aide d'un rhéomètre digital conçu pour caractériser les matériaux pâteux (brevet français 85-14-870). L'appareillage consiste en un palpeur mobile cylindrique 
Tableau 1. Teneur en eau (\%), note visuelle de déstructuration (de 1 à 5) et pouvoir collant $(N)$ des excretas des dindonneaux de 5 semaines.

\begin{tabular}{|c|c|c|c|}
\hline $\mathrm{N}^{\circ}$ régime ${ }^{111}$ & \begin{tabular}{|l|} 
Teneur \\
en eau
\end{tabular} & $\begin{array}{c}\text { Note visuelle } \\
\text { de destructuration }\end{array}$ & $\begin{array}{l}\text { Pouvoir } \\
\text { collant }\end{array}$ \\
\hline 1 & 78,6 & 3,5 & 1,3 \\
\hline 2 & 80,4 & 3,7 & 1,3 \\
\hline 3 & 78,9 & 2,6 & 1,4 \\
\hline 4 & 76,6 & 2,2 & 0,9 \\
\hline 5 & 80,4 & 3,5 & 1,2 \\
\hline 6 & 76,0 & 1,9 & 0,6 \\
\hline 7 & 75,5 & 1,9 & 0,9 \\
\hline 8 & 77,2 & 1,7 & 0,9 \\
\hline 9 & 72,4 & 2,0 & 1,1 \\
\hline 10 & 75,7 & 3,1 & 1,8 \\
\hline 11 & 75,4 & 2,0 & 0,9 \\
\hline 12 & 80,9 & 3,8 & 2,2 \\
\hline 13 & 79,9 & 3,2 & 1,4 \\
\hline 14 & 77,3 & 2,8 & 1,3 \\
\hline 15 & 75,1 & 1,7 & 0,9 \\
\hline 16 & 75,0 & 1,8 & 0,7 \\
\hline 17 & 77,3 & 2,2 & 1,3 \\
\hline 18 & 80,0 & 4,6 & 1,4 \\
\hline 19 & 76,3 & 2,2 & 0,7 \\
\hline 20 & 77,2 & 2,2 & 1,0 \\
\hline 21 & 80,8 & 3,1 & 0,8 \\
\hline 22 & 76,3 & 2,4 & 1,2 \\
\hline 23 & 83,3 & 4,3 & 1,9 \\
\hline 24 & 78,0 & 2,6 & 1,0 \\
\hline 25 & 81,0 & 3,0 & 1,6 \\
\hline 26 & 77,8 & 2,6 & 0,8 \\
\hline 27 & 78,4 & 2,4 & 0,5 \\
\hline écart-type résiduel & 3,75 & 0,92 & 0,65 \\
\hline Effet des régimes, $P$ & 0,0001 & 0,0001 & 0,0001 \\
\hline
\end{tabular}

(1) Pour la composition chimique des régimes, voir Carré et al (1994).

actionné par un moteur pas à pas et contrôlé par un capteur de force de $50 \mathrm{~N}$ à $0,1 \mathrm{~N}$. L'échantillon $(30 \mathrm{~g})$ de fiente homogénéisé est placé dans une coupelle et compressé par le palpeur à une vitesse de $7,5 \mathrm{~mm} / \mathrm{min}$ jusqu'à ce que la force de compression atteigne $12 \mathrm{~N}$. A ce point, le palpeur est arrêté puis actionné en traction à la même vitesse, avec mesure en continue de la force de traction. La force maximum de traction $(\mathrm{N})$ est enregistrée et présentée comme le pouvoir collant de l'échantillon.

Tableau 2. Equations ( $n=27)$ exprimant la note visuelle de déstructuration des excretas (de 1 à $5 ; Y_{1}$ ) et leur pouvoir collant $\left(N ; Y_{2}\right)$ en fonction de la viscosité utile réelle $\left(X_{1}\right)$, de la teneur en parois végétales insolubles dans l'eau $\left(X_{2}\right)$ et de l'" eau retenue par les parois " $\left(X_{3}\right)$, mesurées dans les régimes.

\begin{tabular}{|lll|}
$\mathrm{Y}_{1}=0,724 \mathrm{X}_{1}+1,36$ & Ecart-type résiduel $=0,378$ & $\mathrm{R}^{2}=0,775$ \\
$\mathrm{Y}_{1}=0,706 \mathrm{X}_{1}-0,136 \mathrm{X}_{2}+3,28$ & Ecart-type résiduel $=0,310$ & $\mathrm{R}^{2}=0,850$ \\
$\mathrm{Y}_{1}=0,683 \mathrm{X}_{1}-0,00151 \mathrm{X}_{3}+3,11$ & Ecart-type résiduel $=0,340$ & $\mathrm{R}^{2}=0,819$ \\
$\mathrm{Y}_{2}=0,199 \mathrm{X}_{1}-0,00153 \mathrm{X}_{3}+2,48$ & Ecart-type résiduel $=0,292$ & $\mathrm{R}^{2}=0,485$ \\
\hline
\end{tabular}

Les valeurs de $X_{1}, X_{2}$ et $X_{3}$ ont été présentées dans un article précédent (Carré et al 1994). $0,83 \leqslant \mathrm{X}_{1} \leqslant 4,49\left(\log (\eta \mathrm{r}) \cdot \mathrm{g}^{-1} \cdot \mathrm{ml}\right)$

$11,2 \leqslant X_{2} \leqslant 17,1 \%$

$783 \leqslant \mathrm{X}_{3} \leqslant 1346 \mathrm{~g} / \mathrm{kg}$

\section{Résultats et discussion}

Les valeurs individuelles des notes de déstructuration et du pouvoir collant des excretas sont positivement corrélées entre elles $\left(R^{2}=0,261\right)$ et sont chacune positivement corrélées à la teneur en eau des excre$\operatorname{tas}\left(R^{2}=0,553\right.$ et $R^{2}=0,227$, respectivement). La teneur en eau des excretas dépend positivement de la "Viscosité Utile Réelle " (VUR) $(P=0,0001)$ et négativement, de la teneur en parois végétales (PAR) des régimes $(P=0,0231)$, un point supplémentaire de pourcentage de PAR dans les régimes entrainant en moyenne une réduction de un demi-point de pourcentage d'eau dans les excretas. Les parois végétales interviennent probablement ici comme des diluants de l'eau excrétée.

L'analyse de variance montre un effet significatif des régimes (tableau 1) sur le pourcentage d'eau dans les excretas, la note de déstructuration et le pouvoir collant des excretas.

Les moyennes par régime ont été analysées par régression linéaire multiple en utilisant, pour variables explicatives, les caractéristiques physico-chimiques des régimes présentées dans l'article précédent (Carré et al 1994). Les régressions les plus précises sont présentées au tableau 2. La variable VUR intervient dans toutes les équations, ce qui n'est pas surprenant puisque les variables à expliquer sont liées à la teneur en eau des excretas qui est elle-même dépendante de la

Tableau 3. Teneur en parois insolubles dans l'eau (PAR) et valeur de leur pouvoir de rétention d'eau, mesurées pour 26 matières premières.

\begin{tabular}{|l|r|r|}
\hline & PAR (\% MS) & $\begin{array}{r}\text { Rétention } \\
\text { d'eau (g/g) }\end{array}$ \\
\hline Mais & 10,0 & 8,6 \\
Blé & 11,0 & 8,6 \\
Orge & 18,0 & 7,6 \\
Triticale & 13,3 & 9,5 \\
Sorgho 0 tanin & 8,0 & 9,3 \\
Avoine & 31,1 & 4,3 \\
Seigle & 13,6 & 10,8 \\
Manioc & 5,8 & 18,9 \\
Pois & 13,7 & 12,7 \\
Féverole & 16,5 & 10,3 \\
Graine entière Soja toasté & 16,8 & 17,1 \\
Graine entière Tournesol & 27,1 & 7,3 \\
Graine entière Colza 00 & 20,9 & 10,4 \\
Tourteau Soja S (52,6 \% PB/MS) & 18,9 & 16,0 \\
Tourteau Soja B (51,5\% PB/MS) & 19,8 & 13,3 \\
Tourteau Tournesol & 43,3 & 10,1 \\
Tourteau Colza & 35,8 & 10,9 \\
Gluten de maïs & 1,5 & 3,5 \\
Remoulage de blé & 18,5 & 10,1 \\
Corn gluten feed & 36,5 & 5,9 \\
Corn distiller & 32,4 & 6,9 \\
Luzerne & 38,6 & 17,8 \\
P x 1 & 7,1 & 9,6 \\
Coques de soja & 56,8 & 7,8 \\
Coques de tournesol & 81,6 & 5,3 \\
Coques de colza 00 & 66,0 & 5,7 \\
\hline
\end{tabular}


Figure 1. Prédiction du pouvoir collant des excretas de dindonneaux de cinq semaines par la viscosité utile réelle (VUR, $\left.\mathrm{g}^{-1} \cdot \mathrm{ml}\right)$ et l'eau retenue par les parois végétales (EauPAR, $\mathrm{g} / \mathrm{kg}$ ), mesurées sur les régimes.

Pouvoir collant $(\mathrm{N})$ (Moyenne par régime, 8 animaux/régime)

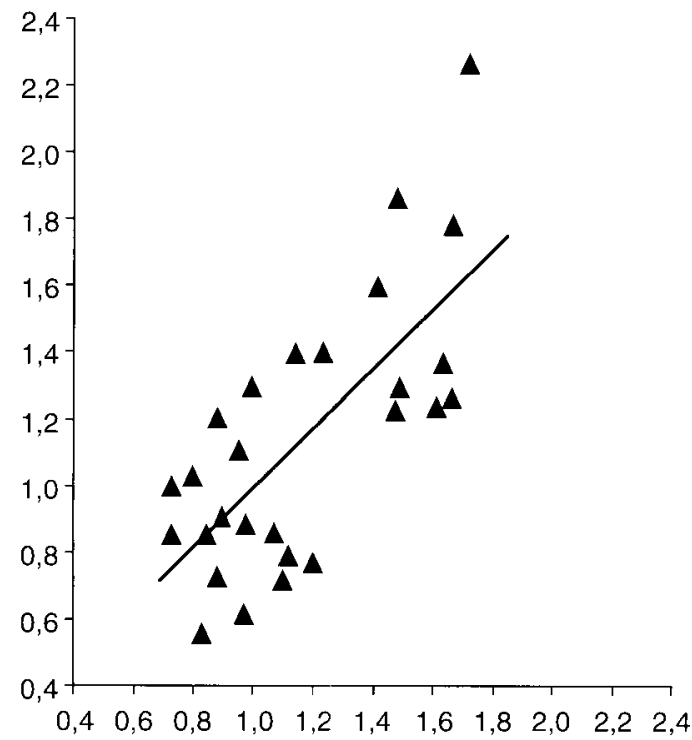

Valeurs prédites d'après l'analyse des régimes:

$\mathrm{Y}=0,20$ VUR $-0,00153$ EauPAR $+2,5 \quad R^{2}=0,485$

VUR. Les teneurs en parois végétales et en " eau retenue par les parois » (EauPAR) interviennent également mais dans le sens inverse de la VUR (tableau 2, figure 1). En d'autres termes, plus les aliments renferment de parois végétales ou "d'eau retenue par les parois ", plus les fientes sont sèches et paraissent cohérentes, et plus leur pouvoir collant est faible. Ce résultat est relativement logique compte tenu du fait que les parois végétales ne sont pas digérées par le poulet (Carré et al 1990), elles conservent donc leurs propriétés physiques dans les excretas. Vis-àvis de l'eau, ces parois végétales vont agir comme un buvard et donc diminuer l'eau circulante dans les excretas qui auront ainsi un aspect moins liquéfié, et un pouvoir collant plus faible du fait de la réduction de la surface de contact assurée par l'eau circulante.

Il est probable que l'état de l'eau dans les excretas peut aussi avoir une influence sur la répartition spatiale de l'humidité dans l'élevage. En effet, si l'eau excrétée est peu liée dans les excretas du fait d'une faible rétention pariétale, elle va avoir tendance à couler au fond de la litière ou à s'évaporer facilement. A l'inverse, des excretas où l'effet "buvard " des parois serait important vont probablement retenir l'eau en surface de litière d'une manière plus importante.

L'impact probable des parois végétales et de leur rétention d'eau sur la qualité des litières mérite un examen précis des matières premières entrant dans la confection des régimes. Le tableau 3 présente les résultats des mesures effectuées sur 26 matières pre- mières. Les parois végétales dont les rétentions d'eau sont les plus élevées se rencontrent dans les dicotylédones, probablement du fait de la présence de substances pectiques dans ces parois (Carré et Brillouet 1986). Le manioc possède les parois dont le pouvoir de rétention est le plus élevé, mais sa teneur en parois végétales est très faible, ainsi la quantité "d'eau retenue par les parois"ne dépasse celle du blé que de $16 \%$. En fait, les quantités "d'eau retenue par les parois" vont être les plus élevées, en ordre décroissant, pour la luzerne, les tourteaux de tournesol, colza et soja, le corn gluten feed, le remoulage de blé et le pois. Par rapport à ces dernières matières premières, les céréales usuelles maïs et blé se trouvent à des niveaux beaucoup plus faibles.

L'excellente additivité de la mesure de "l'eau retenue par les parois" permet au formulateur d'utiliser ce paramètre. Le formulateur doit cependant tenir compte du traitement technologique appliqué à l'aliment. En effet, les 27 aliments granulés utilisés dans l'expérience nutritionnelle présentaient des différences entre les valeurs calculées par additivité et les valeurs mesurées, qui variaient en fonction de la température en sortie de presse (figure 2). En d'autres termes, les fortes températures de granulation réduisent le pouvoir de rétention d'eau des parois végétales, cette diminution atteignant environ $20 \%$ des valeurs d'origine pour une température de $100^{\circ} \mathrm{C}$ en sortie de filière. Cet effet s'explique probablement par la modification des espaces entre les polysaccharides des parois due à l'élimination des molécules d'eau, provoquée par la compression et l'évaporation.

Figure 2. Relation entre «'eau retenue par les parois " (EauPAR) mesurée, la valeur calculée par additivité $\left(X_{1}\right)$ et la température en sortie de presse $\left(X_{2}\right)$.

EauPAR $(\mathrm{g} / \mathrm{kg})$ mesurée des régimes granulés

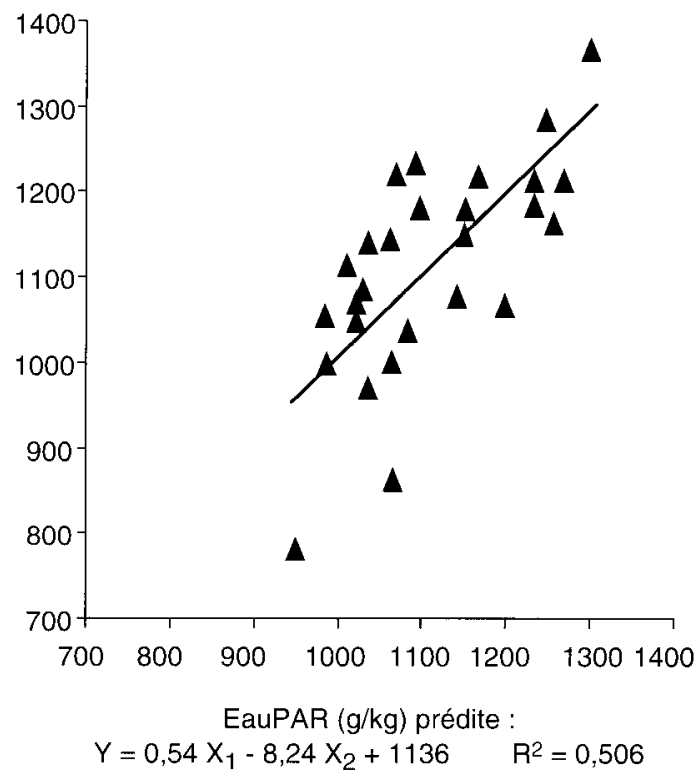


Si les quantités d'eau excrétées et les caractéristiques physiques des excretas peuvent jouer un rôle dans la qualité des litières, il ne faut pas pour autant minimiser l'effet du support de la litière (paille, copeaux, etc.) et du sol qui, eux aussi, possèdent leurs propres propriétés de rétention d'eau.

\section{Remerciements}

La présente étude a été réalisée avec le soutien financier du Fonds SYPRAM (fonds constitués par les adhérents de l'Association pour le Maintien de l'Elevage en Bretagne (AMEB), du Syndicat National des Industriels de la Nutrition Animale (SNIA), et de la Fédération Nationale des Coopératives de Production et d'Alimentation Animales (SYNCOPAC)) et du CRITT Valicentre (Projet SYPRAM n"11, Mars 1992 - Mars 1994), commanditaires du projet.

Nous remercions les représentants des firmes et coopératives de fabrication d'aliment participant au Fonds SYPRAM pour leurs rôles de conseil. Nous adressons également nos remerciements à D. Guibert (L.T.A.N., INRA de Nantes) pour sa participation dans les travaux concernant l'évaluation des propriétés de collage des excretas. Toute reproduction totale ou partielle doit faire mention des commanditaires de l'étude et de l'origine de son financement.

\section{Références bibliographiques}

Carré B., Brillouet J.M., 1986. Yield and composition of cell wall residues isolated from various feedstuffs used for non-ruminant farm animals. J. Sci. Food Agric., 37, 341-351.

Carré B., Derouet L., Leclercq B., 1990. Digestibility of cell wall polysaccharides from wheat (bran or whole grain), soybean and white lupin meal in coc- kerels, Muscovy ducks and rats. Poult. Sci., 69, 623633.

Carré B., Gomez J., Melcion J.P., Giboulot B., 1994. La viscosité des aliments destinés à l'aviculture. Utilisation pour prédire la consommation et l'excrétion d'eau. INRA Prod. Anim., 7, 369-379.

Abstract

\begin{abstract}
Litter quality in poultry production. Relationship between the feeds and the physical characteristics of excreta.

The current paper contains further results of a previous experiment in which predictions of water consumption and excretion were established for meat turkey-poults. The results, here, concern the prediction of the physical aspect of excreta. The excreta of turkey poults fed on 27 different pelleted diets were analyzed for their sticky properties using a rheometer designed for pastes. Visual scores for structural defects were also assigned to the excreta. The sticking level and the visual score were positively correlated $(\mathrm{R} 2=0.26 ; \mathrm{n}=216)$ between themselves, and were each positively correlated to the water content of the excreta $(R 2=0.23$ and $R 2=0.55$, respectively; $n=216$ ). These measurements (mean values per diet) were related to the diet analyse using multiple linear regressions. The
\end{abstract}

best equations were obtained with the variables "real applied viscosity" (RAV) and "cell wall retained water" (Watercw). The coefficients of RAV and Watercw variables were positive and negative, respectively. The $R 2$ values of regression lines were 0.485 and 0.819 for the sticking level and the visual score, respectively. The water retention capacity of cell walls from diets depended on those of the raw materials introduced in diets and on the outlet pelleting temperature. The highest temperatures led to a reduction in the water retention capacity of cell walls. The latter capacities varied in a wide range (1: 4 minimum : maximum ratio) between the different raw materials.

CARRÉ B., DE MONREDON F., MELCION J.P., GOMEZ J., 1995. Qualité de la litière en aviculture. Aliments et caractéristiques physiques des excretas. INRA Prod. Anim., 8 (5), 331-334. 